of other machine components to assess overall machine simultaneity.

\section{Conclusions}

This new switching technique has proven to be an effective and inexpensive method of reducing prepulse voltages on high voltage accelerators. The switches easily allow two or more PFL's to be connected in parallel for increased power density. By paralleling switches, the effective jitter from a module with ten parallel switches is only 40 percent of the jitter of an individual switch. For short pulse accelerators, the output rise time increases rapidly with increasing charge voltage and charge time.

\section{ACKNOWLEDGMENT}

The authors wish to thank J. F. Seamen and W. B. S. Moore for their contributions in the fabrication, installation, and testing of the switches, and R. M. Mason, Jr., of K Tech Corporation, for his assistance in the statistics calculations.

\section{REFERENCES}

[1] J. C. Martin, "Multichannel gaps," Internal Rep. SSWA/JCM/703/ 27, AWRE, Aldermaston, England, 1970.

[2] J. K. Burton, D. Conte, W. H. Lupton, J. D. Shipman, Jr., and I. M. Vitkovitsky, in Sth Symp. Eng. Problems Fusion Res., Princeton, NJ, 1973, pp. 679-683.

[3] J. P. VanDevender and T. H. Martin, IEEE Trans. Nucl. Sci., vol. NS-22, p. 979, 1975

[4] B. A. Demidov, M. V. Ivkin, V. A. Petrov, E. A. Smirnov, and S. D. Fanchenko, in Proc. 2nd Int. Topical Conf. High Power Electron and Ion Beam Res. and Tech., Ithaca, NY, 1977, p. 771.

[5] J. C. Martin, "Nanosecond pulse techniques," Internal Rep. SSWA/JCM/704/49, AWRE, Aldermaston, England, 1970

[6] J. P. VanDevender, J. Appl. Phys., vol. 49, p. 2616, 1978.

[7] T. H. Martin, D. L. Johnson, and D. H. McDaniel, in Proc. 2nd Int. Topical Conf. High Power Electron and Ion Beam Res. and Tech., Ithaca, NY, 1977, p. 807

\title{
Experiments on Electrical Breakdown in Water in the Microsecond Regime
}

\author{
DAVID B. FENNEMAN AND RONALD J. GRIPSHOVER
}

\begin{abstract}
This paper presents experimental results from research on electrical breakdown in water carried out at NSWC/DL. The experimental apparatus is described in some detail. Results from approximately two thousand tests are presented. Breakdown events were observed predominantly in the 2-10- $\mu$ s regime for applied fields in the range $150-500 \mathrm{kV} / \mathrm{cm}$. The shot-to-shot variation of breakdown time intrinsic to the phenomena requires statistical measures of performance. The performance of four electrode materials-copper, brass, stainless steel, and aluminum-is presented. The first three performed similarly ; aluminum was significantly different.
\end{abstract}

\section{INTRODUCTION}

W ATER, BECAUSE of its high dielectric constant, selfrepairability, low cost, and ease of handling is commonly used as the intermediate energy storage medium in pulsed power devices. Large high-energy high-power machines frequently have a capacitor or pulse forming line charged in the multimicrosecond regime. The water must not suffer electrical breakdown during this charging time. These considerations have led the pulsed power group at NSWC/DL to

Manuscript received February 14, 1980; revised April 17, 1980.

The authors are with the Naval Surface Weapons Center, Dahlgren, VA 22448 . pursue research on this topic. The goals of the effort are to provide empirical performance comparisons in order to establish design-tradeoff rationale, and to provide experimental evidence to test various theories of breakdown.

In the regime investigated in this work, the process of electrical breakdown has wide (apparently) statistical variation. It is not uncommon for identical tests to differ by a factor of two in time of breakdown. To obtain reliable measures of performance under these conditions requires large numbers of tests and good control on all process variables. These considerations underlie the experimental approach.

\section{Apparatus}

The test apparatus built at NSWC/DL explicitly for water breakdown research consists of three components (refer to Fig. (1)): a water conditioning system, an electrical system, and the test cell.

The water conditioning system was designed to provide water which could be well characterized. It consists of a) a pump of $>4 \mathrm{gal} / \mathrm{min}$ capacity, b) a mixed bed deionizer, c) a deaeration column, d) a heat bath to maintain temperature, and e) an ultraviolet sterilizer to suppress algae growth. This 


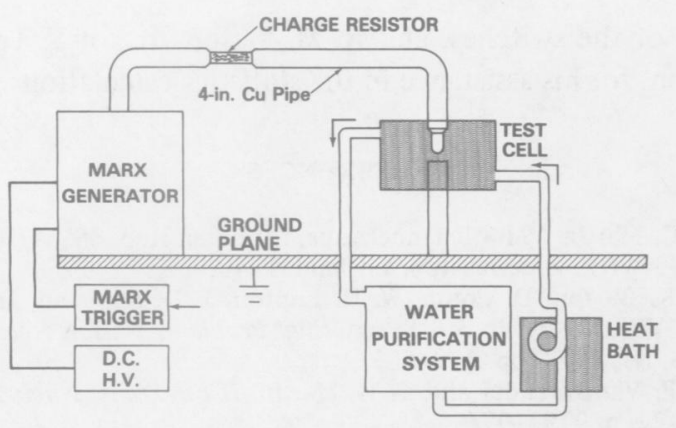

Fig. 1. Water breakdown experiment.

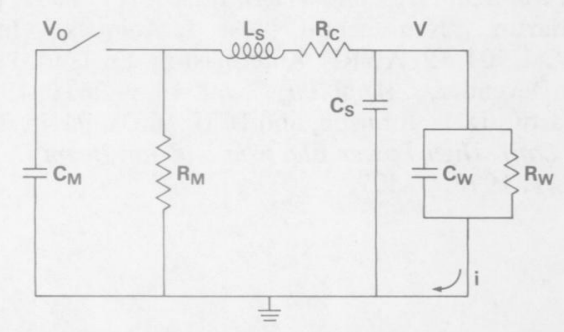

Fig. 2. Electrical circuit. $C_{m}$ (Marx capacitance) $=22 \mathrm{nF} ; R_{m}$ (Marx internal resistance $)=900 \Omega ; L_{s}($ stray inductance $) \simeq 4 \mu \mathrm{H} ; C_{S}($ stray capacitance $) \simeq 0.1 \mathrm{nF} ; R_{c}$ (charge resistor) $\simeq 1.7-4.0 \mathrm{k} \Omega ; C_{w}$ (water capacitance $) \simeq 0.4-1.2 \mathrm{nF} ; R_{w}$ (water resistance $)>300 \mathrm{k} \Omega$.

last item is used only intermittently and may not be necessary. Resistivity probes measure the resistivity of the water at the outlet of the deionizer and at the outlet of the test cell. Temperature is measured by thermistor probes located at the outlet of the heat bath, at the outlet of the test cell, and in the deaeration column. The pressure in the deaeration column is maintained by a vacuum pump, protected from water vapor fouling by a trap cooled by an alcohol-dry ice slurry. The pressure is measured by a mercury manometer. The water is conditioned for about $3 \mathrm{~h}$ before testing, and continually during testing. All told, about 40 gal of treated water are continually circulated. It takes about an hour to bring the resistivity of the water to above $18 \mathrm{M} \Omega \cdot \mathrm{cm}\left(25^{\circ} \mathrm{C}\right)$ from the $2-3-\mathrm{M} \Omega \cdot \mathrm{cm}$ value the water degrades to overnight. The resistivity obtained in the system is at or near the ultimate value for water. Success in obtaining such high values is ascribed to flowing continually above $2.5 \mathrm{gal} / \mathrm{min}$ and to the fact that with the exception of the test electrodes, the copper coils of the heat bath, and the small area of the stainless steel probes, the water touches no metal or glass. All pipes and valves are hard polyvinyl chloride (PVC), the pump has a nitrate impeller, and the deaeration column is Plexiglas. At equilibrium at temperature $T$, the percent deaeration is computed as

$$
\text { Percent deaeration }=100 \times\left(1-\frac{p-P_{\mathrm{H}_{2} \mathrm{O}}(T)}{760}\right)
$$

where $p=$ pressure in deaeration column (torr), and $P_{\mathrm{H}_{2} \mathrm{O}}(T)=$ water vapor pressure (torr).

The circuit of the electrical system is shown in Fig. 2. The voltage source is a 10 -stage Marx generator capable of $500-\mathrm{kV}$ maximum, whose erection time is a couple of hundred nanoseconds. The Marx charges the water test cell with either polarity through a copper sulphate charging resistor. The

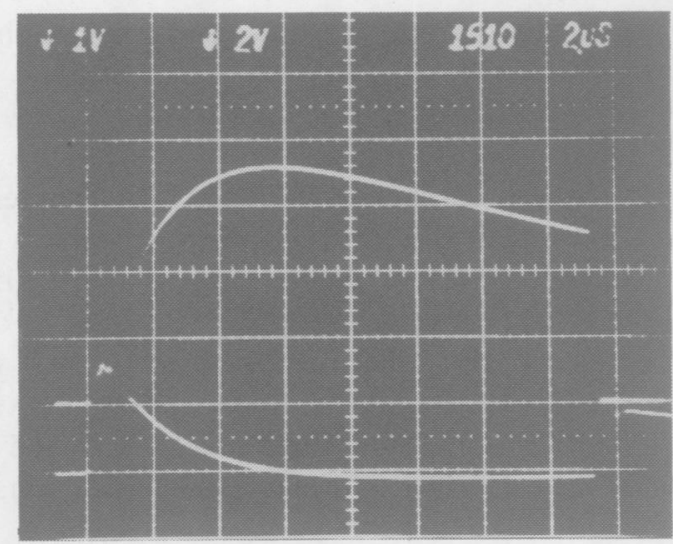

Fig. 3. Experimental waveforms. Top trace $V(t), 40 \mathrm{kV} /$ div. Bottom trace $i(t), 40 \mathrm{~A} /$ div. The number to the right of center in the display is the breakdown time in tens of nanoseconds $(15.1 \mu \mathrm{s})$.

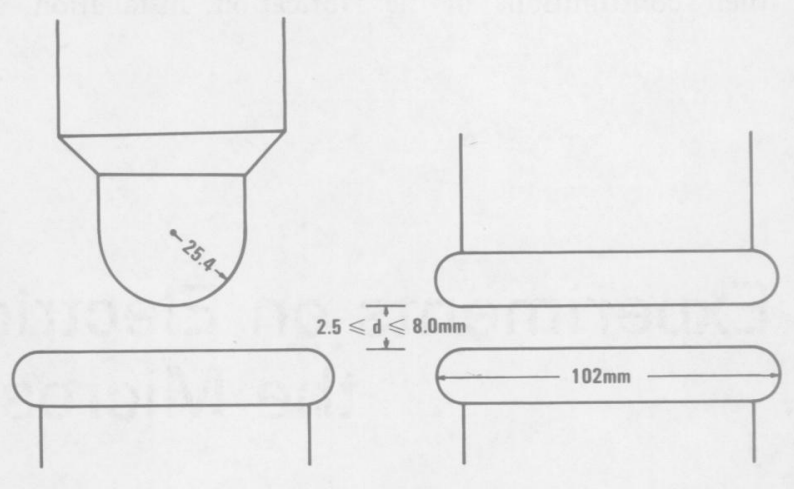

Fig. 4. Electrode geometries. All dimensions are in millimeters.

voltage also bleeds through a Marx internal resistance of approximately $900 \Omega$. Circuit inductance is unimportant and the voltage across the water is closely given by

$$
V(t)=0.71 V_{0}\left(e^{-\omega_{1} t}-e^{-\omega_{2} t}\right)
$$

where

$$
\begin{aligned}
V_{0} & =\text { erected Marx voltage } \\
1 / \omega_{1} & \simeq R_{m} C_{m} \simeq 20 \mu \mathrm{s} \\
1 / \omega_{2} & \simeq R_{c}\left(C_{w}+C_{s}\right) \simeq 2.0 \mu \mathrm{s} .
\end{aligned}
$$

The voltage is measured by a copper sulphate dividing resistor, the current is measured by a Rogowski coil. The observed voltage and current waveforms agree with computer modeling (which takes into account temperature and gap size effects) to the resolution of the oscilloscope traces. The waveform repeatability from shot to shot is within two percent. Breakdown time is also measured by counting a 100 $\mathrm{MHz}$ clock signal gated by the voltage signal. All are recorded on a Tektronix Model 7844 Dual Beam Oscilloscope. Fig. 3 shows a sample test trace.

The test cell is a Plexiglas box $0.5 \mathrm{~m} \times 0.5 \mathrm{~m} \times 0.36 \mathrm{~m}$ which holds the test electrodes. The electrodes are thus over $0.1 \mathrm{~m}$ away from any solid dielectric interfaces which might contribute to field anisotropies or cause spurious breakdown paths. Two electrode geometries are used; hemisphericalplane and planar. These are sketched in Fig. 4. The hemispherical-plane electrodes are copper. Four sets of electrodes, 
made of copper, brass, stainless steel, and aluminum are in the planar configuration. The final surfacing for all is done by glass bead blasting (Zero-Blast-N-Peen, size 801, Mil-G 9954A). The scale of granularity imposed on the electrode surfaces is on the order of the size of the glass beads, about $125 \mu \mathrm{m}$. This surfacing technique is chosen only because it produces a wellcharacterized reproducible surface.

The gap is visible during testing and the spacing is measurable to $0.03 \mathrm{~mm}$ by a cathetometer. The planar electrodes are kept parallel to within $0.3 \mathrm{~mm}$ which is typically 5 percent of the gap spacing. One of the coordinates of the breakdown position is monitored visually by use of the cathetometer (i.e., the azimuthal coordinate with respect to the cathetometer).

\section{Performance Criteria}

Since the pioneering work of J. C. Martin and others at Aldermaston on water breakdown, it has become customary to express the electrical stress and the maximum duration for which water will hold the stress in the form of a functional relation [1]

$$
E_{\max } t_{\mathrm{eff}}^{1 / 3}=M / A^{1 / 10} .
$$

$E_{\max }$ is the maximum electric field in the water, $t_{\text {eff }}$ is the effective time (defined as the duration for which the field exceeds 63-percent $E_{\max }$ ), $A$ is the area of the electrodes which experience 90 percent or more of the field. For $E$ in $\mathrm{MV} / \mathrm{cm}, t_{\text {eff }}$ in $\mu \mathrm{s}, A$ in $\mathrm{cm}^{2}, M$ is a "constant" which has values reported to range between 0.3 and 0.6 [2], [3]. $M$ might be expected to depend on the quality of water (purity percent deaeration, temperature, etc.) and on the electrodes (geometry, surface finish, material, etc.). Thus the value of $M$ can be used as a figure of merit for a water capacitor system. The larger $M$ is, the better.

Assuming (2), $M$ can be obtained experimentally by measuring the field waveform applied to the system and monitoring the time to breakdown. $t_{\text {eff }}$ is then defined as

$$
t_{\text {eff }}=t_{b}-t_{0.63} \text {. }
$$

$t_{b}$ is the breakdown time measured from onset of the field and $t_{0.63}$ is the time at which the field first exceeds 63 percent of the maximum field achieved. In this way $M$ is only based on tests in which the water actually broke down.

Since experiments show wide shot-to-shot variation, $M$ is estimated by averaging over $N$ shots

$$
\langle M\rangle_{N} \equiv \frac{1}{N} \sum_{i}^{N} E_{\max _{i}}\left(t_{\mathrm{eff}_{i}}\right)^{1 / 3} A^{1 / 10} .
$$

Trends are examined by computing running averages of an index $j$, thus

$$
\left\langle M_{j}\right\rangle_{20}=\frac{1}{20} \sum_{i=j}^{i=j+19} M_{i} .
$$

During the initial testing, it was noted that for lower voltages breakdown often did not occur. Accordingly, a second measure of breakdown, called "breakdown probability" is defined to reflect this threshold character. Simply stated, if $n$ tests with identical waveforms are performed and $b(\leqslant n)$ of them
TABLE I

Materials Study, Grand Means of All Breakdowns ${ }^{a}$

\begin{tabular}{ll}
\hline \hline ELECTRODE & $\langle M\rangle \pm 100 \frac{\sigma(M)}{\langle M\rangle}$ \\
HEMISPHERICAL $\left(A=3 \mathrm{~cm}^{2}\right)$ & \\
CU & $0.564 \pm 13.0 \%$ \\
PLANE $\left(A=81 \mathrm{~cm}^{2}\right)$ & \\
CU & $0.549 \pm 7.3 \%$ \\
STEEL & $0.584 \pm 16.0 \%$ \\
BRASS & $0.580 \pm 10.0 \%$ \\
ALUMINUM & $0.408 \pm 16.0 \%$ \\
\hline
\end{tabular}

a The hemisphere was stressed positive, when stressed negative, $\langle M\rangle$ is 5 percent higher $(0.594)$.

suffer breakdown, the ratio $b / n$ is called the breakdown probability regardless of when the breakdown occurred.

\section{Testing Procedure and Results}

Experimental research on breakdown phenomena is subject to a wide variety of influences, the importance of each difficult to assess $a$ priori. The best that can be done is to maintain the environment and testing procedure as constant as possible. Knowledge of these factors is required to assess the applicability of results. The test results reported here were obtained over a period of about six months. The electrodes were tested in the order: hemispherical copper, planar steel, planar aluminum, planar brass, and planar copper. The temperature for the hemispherical plane tests were $19 \pm 2{ }^{\circ} \mathrm{C}$; for all the planar tests, it was $16 \pm 1^{\circ} \mathrm{C}$. The hemispherical-plane geometry was more extensively tested and sat in deaerated water over three months. This was the first set of tests and there was trepidation that the data might be colored by the fact that this sequence was used to perfect procedures and techniques.

The morning of a typical test day was devoted to circulating the water to: deaerate, bring the resistivity to maximum, and reach thermal equilibrium. Testing was started when deaeration was $>90$ percent and resistivity was within $1 \mathrm{M} \Omega$. $\mathrm{cm}$ of maximum $\left(18.3 \mathrm{M} \Omega \cdot \mathrm{cm}\right.$ corrected to $\left.25^{\circ} \mathrm{C}\right)$. Once testing was started, shots were on the order of 2 min apart (this is the charging time of the Marx). In this interval the water volume between electrodes is changed hundreds of times. Usually 10 shots at a given Marx setting were made. 50 to 100 shots could be made during the afternoon.

Test results for all tests are summarized in Table I. The close agreement between the hemispherical copper $\left(A=3 \mathrm{~cm}^{2}\right)$ and the planar copper $\left(A=81 \mathrm{~cm}^{2}\right)$ removes some of the trepidation previously mentioned. It is to be emphasized that the variations are not due to measurement uncertainties, but inherent in the breakdown process.

From the beginning there was concern over aging. Aging (i.e., the change in breakdown character with time, or number of breakdowns suffered) based on two mechanisms could be postulated. One mechanism is chemical reaction with water, the other is surface field anisotropies due to pitting and scarring from repeated tests. This is studied by arranging the data in order taken and computing running averages of $M$. The results for the four sets of planar electrodes are shown in Fig. 5. All except aluminum show no clear trend. Aluminum requires further discussion, given below. 


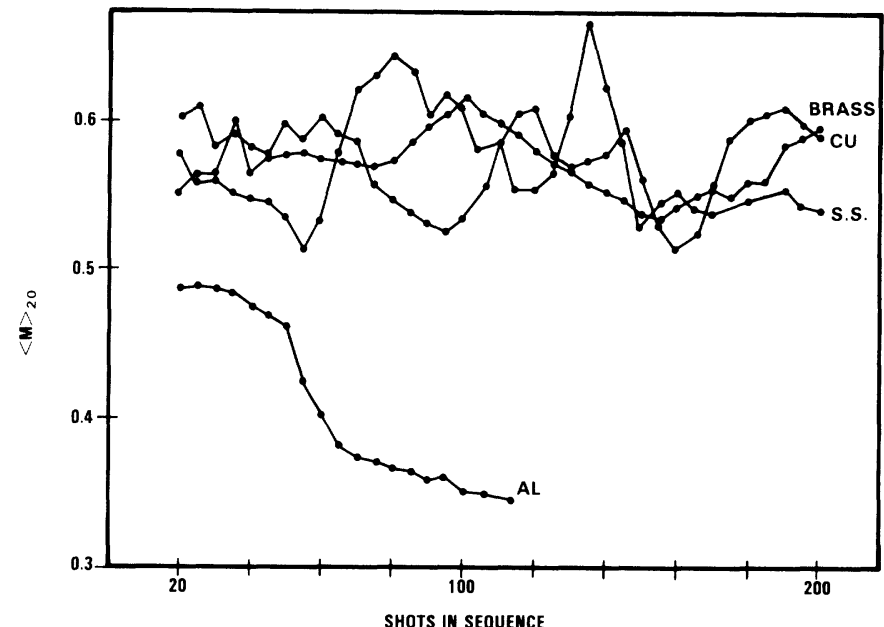

Fig. 5. Aging study. $\langle M\rangle$ for 20 tests as a function of the shot sequence number.

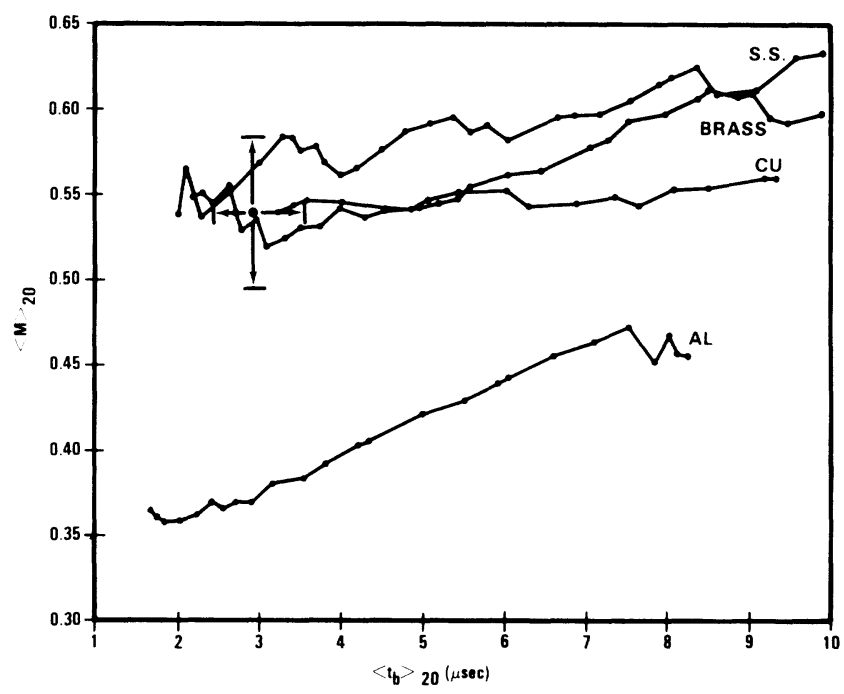

Fig. 6. Variation of $\langle M\rangle_{20}$ with mean time to breakdown. If $M$ as given in (2) was a universal measure of the field-time breakdown relationship, it would be expected to remain constant.

Another question concerned the range of validity of (2) for long breakdown times $\left(t_{\text {eff }} \gg 1 \mu \mathrm{s}\right)$. This was studied by arranging all breakdown data in order of $E_{\max }$ and computing running averages of $M$ and time to breakdown. Results are displayed in Fig. 6. Here a clear trend for $\langle M\rangle_{20}$ to increase with increasing $\left\langle t_{b}\right\rangle_{20}$ is seen. This suggests a weaker than $t^{1 / 3}$ time dependence with increasing breakdown time.

To check the reproducibility of the results, after all testing was completed, the stainless steel electrodes were resurfaced and 44 shots all with the identical waveform were performed. This data in Fig. 6 as the single point with error lines whose extension are the root-mean-square variance.

In comparing Figs. 5 and 6, conclusions can only be drawn if the shots in sequence bear no overall correlation to $E_{\max }$. In reviewing the data, this was found to be true for all except, unfortunately, the aluminum data. Test sequence data for aluminum was correlated to $E_{\max }$ (i.e., each aluminum data point was taken at the same or higher breakdown field than the previous data point). Hence it is not possible to ascribe the trend displayed with certainty purely to nonconstancy of $M$ or aging, or both.

The results of threshold testing are shown in Fig. 7. The

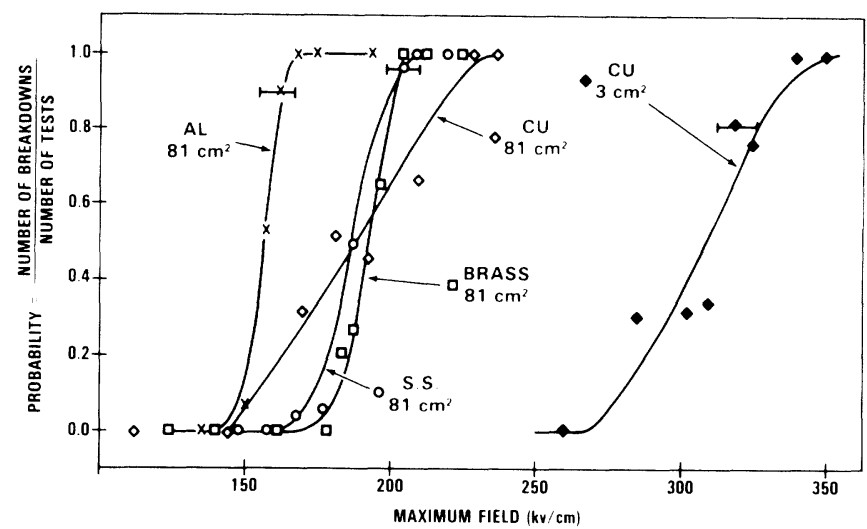

Fig. 7. Results of threshold testing.

transition from no breakdowns to 100 -percent breakdown is fairly sharp. Once again, experimental vicissitudes need discussion. If these tests are performed starting at a low field and completed by monotonically increasing the field until 100 . percent breakdowns occur, a fairly smooth and sharp plot of breakdown probability will result. Similarly, if one starts with a high field and monotonically decreases the field, smooth data result. However, the plots will not interlace. Increasing sequence probabilities always seem to be at higher fields than when data is taken in decreasing fashion. The points plotted are combinations of both methods. This effect is generalized by stating the experimental observation that the probability of breakdown on the $n$th test depends on whether the $n$-1st test broke down, which is to say each test is not a Bernoulli chance. This effect, which is difficult to quantify, was explored in a qualitative way. It was established that, following application of a high stress, a lower stressed test would probably break down. Quite a bit of effort was expended to determine if there was a correlation between breakdown sites. By means of the cathetometer, the breakdown position was measured for over 400 tests to determine if there was a pattern. None was found, i.e., the measurements indicated that if the $n$th test broke down at position $x$, the probability that any other test would break down at $x$ was small. This was confirmed by posttest examination of the electrodes which revealed a uniform random distribution of breakdown sites.

\section{Summary AND Discussion}

The experiments indicate that bead blasted steel, brass, and copper perform similarly while aluminum performance is significantly lower. This was shown both on the basis of $M$ and breakdown threshold. $M$ for aluminum was about 75 percent of the grand mean $M$ value of the other materials. Similarly the breakdown threshold of aluminum was about 80 percent of the other materials.

The increase of $M$ with $t_{b}$ (Fig. 6) indicates that for longer times, the breakdown field becomes independent of time in a manner similar to the low dielectric constant insulating oils [4], [5]. This is consistent with the work on water breakdown by Miller [6] who recommends for $t_{\text {eff }}>2 \mu \mathrm{s}$ a $t^{1 / 6}$ dependence. Some effort was devoted to examining other field-time relations than (2); such as changing the exponent of the time variation, defining $t_{\text {eff }}$ differently, and so on. For example, in (7) we used a least squares approach to find the best value for a time parameter in place of $t_{0.63}$. While these 
alternate measures could be used to remove the trends shown in Fig. 6, they did not significantly reduce the statistical fluctuations and to avoid confusion, were not presented. Actually, for long times, the threshold field implied by the breakdown probability curves of Fig. 7 is more useful.

Similar speculations concerning the area dependence can be made. The data of Table I show a law of form $A^{1 / 10}$ brings the data for $3 \mathrm{~cm}^{2}$ and $81 \mathrm{~cm}^{2}$ into close agreement. Proceeding another way, one may use the data to solve for the area dependence. This results in an area dependence exponent of 0.108. Miller [6], and VanDevender [2], who worked with larger surfaces, recommend an area exponent 0.06 .

\section{ACKNOWLEDGMENT}

The authors gratefully acknowledge the skill and care of L. W. Hardesty and K. Chilton who constructed the apparatus and assisted in the testing.

\section{REFERENCES}

[1] T. H. Martin, "Pulsed power for fusion," in Proc. Int. Pulsed Power Conf., Lubbock, TX, Nov. 1976, IEEE Cat. No. 76 CH1147-8 Reg 5.

[2] J. P. VanDevender, "Short pulse electrical breakdown strength of $\mathrm{H}_{2} \mathrm{O}$," in Proc. Int. Pulsed Power Conf., Lubbock, TX, Nov. 1976 , IEEE Cat. No. $76 \mathrm{CH} 1147-8$ Reg 5.

[3] I. D. Smith, "Impulse breakdown of deionized water," Pulsed Electrical Power Dielectric Strength Notes, vol. 1, notes 1-23, Air Force Weapons Lab. TR 73-167, note 4, Apr. 1973.

[4] N. S. Rudenko and V. I. Tsvetkov, "Study of the pulse electrical strength of some liquids," Sov. Phys.-Tech. Phys., vol. 9, no. 6, 1964

[5] T. J. Gallagher, Simple Dielectric Liquids. New York: Oxford University Press, 1975.

[6] A. R. Miller, "Low impedance capacitors using pressurized water as a dielectric," in Proc. 5th Symp. on Engineering Problems of Fusion Res., IEEE New York, 1974, p. 471.

[7] D. B. Fenneman and R. J. Gripshover, "Electrical breakdown in water in the microsecond regime," in Digest Tech. Papers, 2nd IEEE Int. Pulsed Power Conf., Lubbock, TX, June 1979, IEEE Cat. No. 79 CH1505-7.

\title{
Effects of Surrounding Medium on Electrically Exploded Aluminum Foil Fuses
}

\author{
T. L. BERGER
}

\begin{abstract}
Flat aluminum foil fuses were exploded electrically by discharging a capacitor bank into a series combination inductance $(\sim 600$ $\mathrm{nH})$ and fuse. The $2.54 \times 2.54 \times 0.0023-\mathrm{cm}$ foils were exploded in a sealed chamber. The time-to-burst (TTB) and fuse voltage characteristics were investigated as a function of the fuse environment. Results are given for foils exploded in various gases and liquids.
\end{abstract}

\section{INTRODUCTION}

$\mathrm{E}$ LECTRICALLY exploded conductors are useful in a wide variety of pulsed power applications. Fast foil current breakers have been used to sharpen current pulses from capacitor banks [1]-[3] and from explosive magnetic flux compression generator-transformer systems [4]-[7]. In addition, fuses have been used as the high speed elements for multiple stage switching in inductive energy storage systems [8]-[9] . Finally, we mention that exploding wires have been used in a number of novel schemes for the purpose of launching hypervelocity projectiles $[10]-[12]$.

Manuscript received February 4, 1980

The author is with the Naval Surface Weapons Center, Dahlgren, VA 22448 .
Despite a wide variety of experimental work, there remains much that is not understood about the electrical explosion of conductors. Edge effects which lead to breakdown, for example, are not well understood. It seems reasonable that breakdown at the edges of the foil is due to corona discharge and explosions due to irregularities which are introduced when the foil is cut. There is some evidence, however, that there may be mechanisms other than corona discharges which lead to edge breakdowns [13]. The effect of volume changes is also not well understood. Although electrical conductivity is known to be relatively sensitive to volume changes, a constant volume approximation is generally used in order to avoid difficult hydrodynamic calculations [14]. Finally, we mention the effects of the surrounding medium on fuse characteristics. It is not clear, for example, what the characteristics of the surrounding medium should be in order to best inhibit electrical breakdown. On the one hand, it is suggested that the surrounding medium should confine the metal vapor in order to inhibit collisionally induced ionization and subsequent breakdown [1]. On the other hand, it has been suggested that heat transfer and chemical reactions with the surrounding medium can inhibit electrical breakdown [15]. 